\title{
ASPECTOS ECOLÓGICOS Y DISTRIBUCION GEOGRÁFICA DE CARRANGENOPHYTAS
}

\author{
MARGARITA DEL R. SALAZAR SÁNCHEZ ${ }^{1}$ \\ RAÚL A. SALAZAR SÁNCHEZ ${ }^{2}$

\begin{abstract}
Recibido el 28 de diciembre de 2011 y aprobado el 5 de
\end{abstract} \\ septiembre de 2012
}

\section{RESUMEN}

Las especies carragenófitas están definidas como macroalgas miembro del Phylum Rodophyta, Clase Rodophyceae, Subclase Florideophycidae, Orden Gigartinales. Las especies de mayor importancia comercial a nivel internacional son de los géneros Betaphycus, Eucheuma y Kappaphycus; y a nivel nacional especies de los géneros Gigartina, Mazzaella y Sarcothalia.

Debido a su importancia comercial, el género Gigartina, ha sido objeto de investigaciones como: Ecología de las poblaciones de las costas estuarinas y abiertas de la especie del noreste del Atlántico; ha sido investigado por Mathieson y Tveter (1976) con relación a las cantidades y calidad de su carragenina. Estudios de las respuestas a fotoperíodos (Guiry, 1984; Guiry et al., 1984) han identificado "ventanas" reproductivas, sugiriendo que las explicaciones de multiplicación vegetativa son cada vez más exclusivas, a diferencia de la producción y gametangial o tetrasporangial, en las poblaciones de las altas latitudes de una especie europea generalizada.

La determinación genética de los patrones de ramificación y límites de tolerancia a la temperatura se han establecido para varias especies de (Guiry et al., 1987.; Buschmann, 2001). La viabilidad del cultivo de especies en sustratos artificiales ha sido evaluada por Mumford y Waaland (1980), y demostró tener potencial comercial. Este género tiene distribución en todo el mundo en mares templados a fríos, sin embargo, muy pocas especies ocurren en las regiones subtropicales. Los centros de diversidad de especies particularmente rica son California, Japón, Nueva Zelanda, Chile y Sudáfrica (Guiry, M.D \& Guiry, G.M. 2011).

\section{PALABRAS CLAVE}

Gigartina, carragenofitas, tetrasporas, carposporas. 


\title{
CARRAGENOPHYTAS ECOLOGICAL ASPECTS AND GEOGRAPHICAL DISTRIBUTION
}

\begin{abstract}
The carragenophytas species are defined as macro-algae, members of the Phylum Rodophyta, Rodophyceae Class, Florideophycidae Subclass, Gigartinales Order. The most important commercial species at the international level are the Betaphycus, Eucheuma and Kappaphycus genera and at the national level the species belonging to the Gigartina, Mazzaella and Sarcothalia genera.
\end{abstract}

Because of its commercial importance, the Gigartina genus has been the object of investigations such as: Ecology of populations in estuaries and open coast in the North-East Atlantic species; it has been studied by Mathieson and Tveter (1976) in relation with its carragenine quantity and quality. Studies about the response to photoperiods (Guiry, 1984; Guiry et al., 1984) have identified reproductive "windows" suggesting that the explanations for vegetative multiplication are more exclusive each time, different from gametangial or tetra-sporangial production in populations of high latitudes from a generalized European species.

Genetic determination of ramification patterns and tolerance limits to temperature has been established for several species (Guiry et al., 1987; Buschmann, 2001). Species cultivation viability in artificial substrates has been evaluated by Mumford y Waaland (1980), and it showed commercial potential. This genus has distribution worldwide in warm and cold seas. However, very few species happen in subtropical regions. The particularly rich species biodiversity centers are California, Japan, New Zealand, Chile y South Africa (Guiry, M.D \& Guiry, G.M. 2011).

\section{KEY WORDS}

Gigartine, carragenophytas, tetra spores, carpospores.

\section{INTRODUCCIÓN}

Las algas del género Gigartina, son algas de color púrpura a rojo intenso, por la dominancia de los pigmentos ficoeritrina y ficocianina, característicos por la presencia de clorofila a (sin clorofila b), $\beta$-caroteno y una serie de xantofilas único (Bold et al., 1985.; El Gamal, 2010)., Son 
gruesas al tacto, de consistencia carnosa, algunas de hábito semicircular a circular en contorno, con numerosos hapterios como rizoides en la parte basal e inferior del tejido que le sirven para adherirse al sustrato. Las frondas alcanzan un tamaño entre los $0.30 \mathrm{~m}$ a $0.60 \mathrm{~cm}$ y en casos muy excepcionales hasta $1.30 \mathrm{~m}$ en diámetro (Figura 1).

Los talos reproductivos son fácilmente reconocidos en apariencia externa. Las plantas tetraspóricas no presentan papilas en la superficie de la lamina y desarrollan soros tetrasporangiales, los cuales se observan como manchas de color oscuro, de forma circular a elipsoidal. Los soros se encuentran inmersos en el talo, desarrollándose en la medula del tejido de las frondas, y distribuyéndose de manera abundante sobre la totalidad de la fronda.

Las plantas masculinas presentan espermatangios superficiales y en láminas lisas. Las frondas femeninas una vez fecundadas desarrollan cistocarpos en papilas, proyectándose notoriamente en la superficie de ésta, con estructuras simples y no ramificadas, alcanzando 4 o 5 cistocarpos en cada papila.

\section{CLASIFICACIÓN}

Tabla 1. Clasificación taxonómica del género Gigartina.

\begin{tabular}{ll}
\hline Eucariota & \\
Reino & Plantae \\
Subreino & Biliphyta \\
Phylum & Rhodophyta \\
Subphylum & Eurhodophytina \\
Clase & Florideophyceae \\
Subclase & Rhodymeniophycidae \\
Orden & Gigartinales \\
Familia & Gigartinaceae \\
Género & Gigartina \\
\hline Fuente: Guiry,M.D. \& Guiry, G.M (2011)
\end{tabular}

\section{ESPECIES}

Las especies Gigartina de mayor tamaño sobrepasan medidas de más de $100 \mathrm{~cm}$ de longitud. Los talos en su mayor parte son erguidos y el rango generalmente foliosos a estrecho pinnados. Algunas especies forman grupos anclados por varios hapterios. En la actualidad, hay 418 especies, de las cuales 35 se han marcado como actualmente aceptadas taxonómicamente (Tabla 2) (Guiry, M.D. \& Guiry, G.M. 2011). 
Tabla 2. Especies de Gigartina

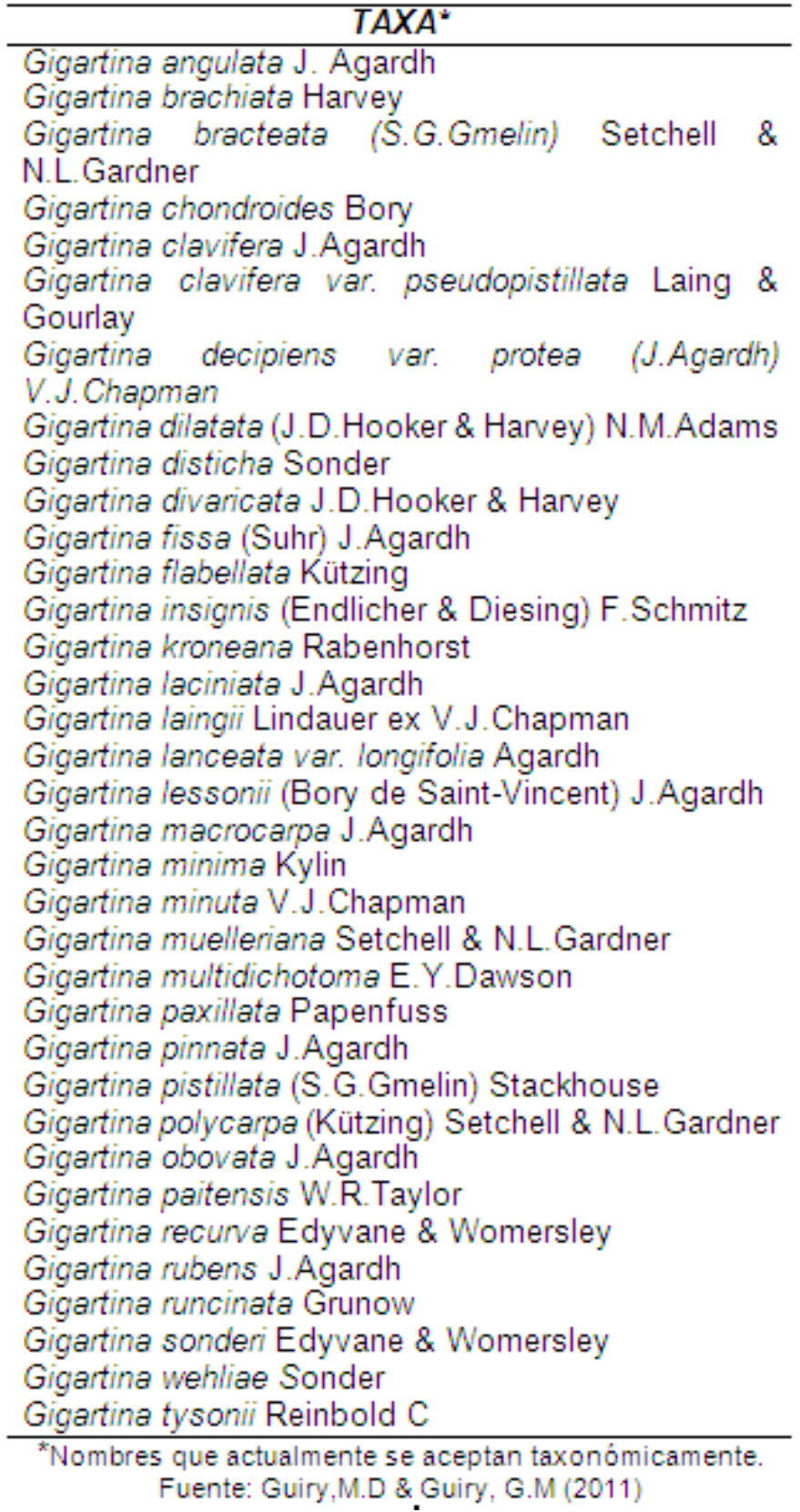



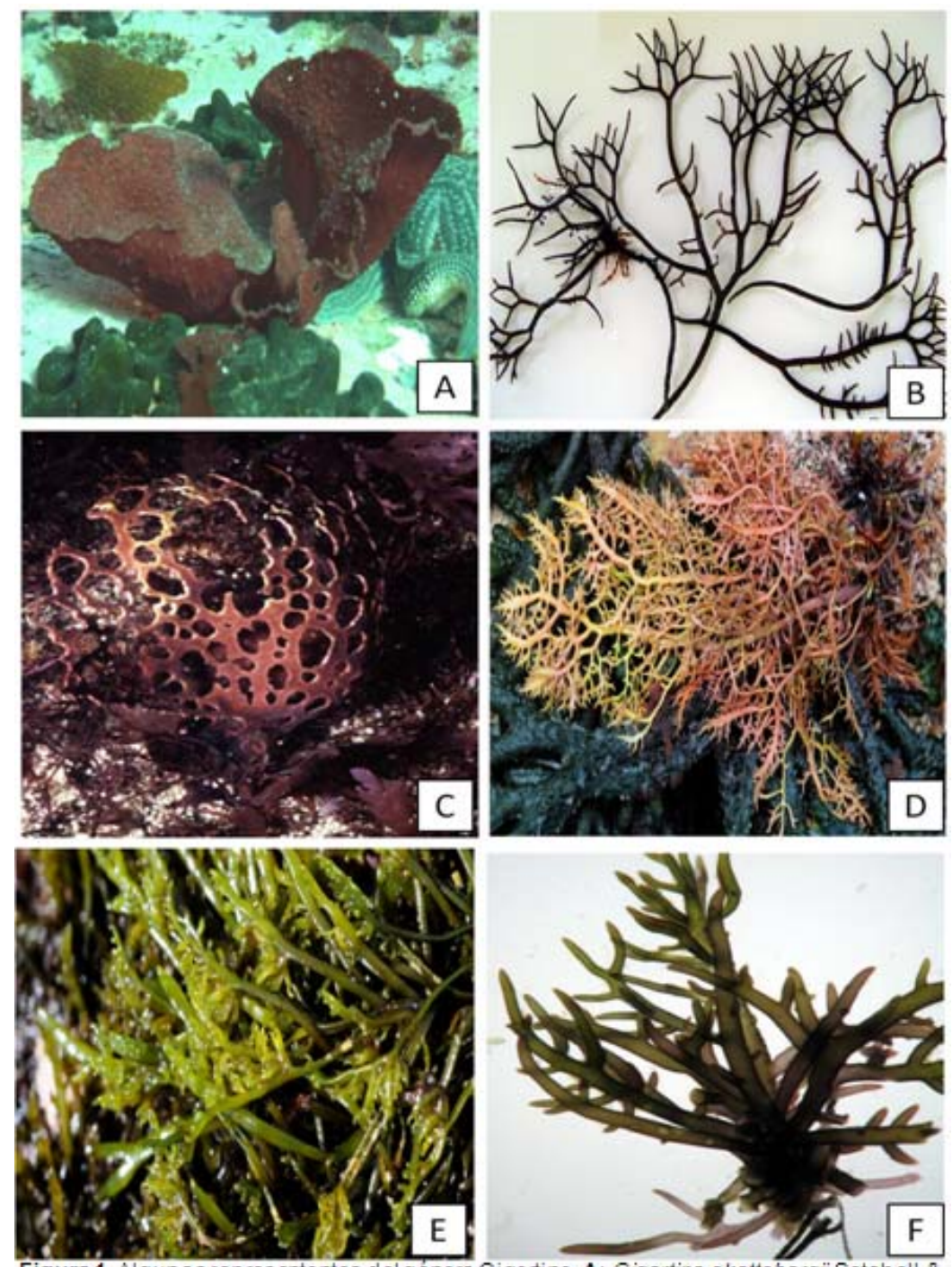

Figura 1. Al gunos representantes del genero Gigartina. A: Gigartina skottsbergï Setchell \& N.L.Gardner., B: G pistillata (S.G.Gmelin) Stackhouse. C: G. bracteata (S.G.Gmelin) Setchell \& N. L.Gardner., D: G disticha Sonder. E: G. minima Kylin., F: G. brachiata Harvey in J.D. Hooker. Fuente: Guiry, M.D. \& Guiry, G.M (2011)

\section{DISTRIBUCIÓN}

Por su importancia económica, con mayor reportaje de investigación se presentará la distribución y hábitos de las tres especies de Gigartina: Gigartina acicularis. Presenta hábitos epilíticos, sobre el litoral inferior y superior en zonas con cierto grado de exposición al oleaje, es una especie tolerante a la cubierta por arena; a veces se encuentra formando un césped denso (Guiry, et al.1983). Esta especie se ha reportado en Irlanda, Europa, en algunas Islas atlánticas como Azores, Islas canarias, Madeira e islas salvajes, también en Norte América en Florida y Norte de Carolina; en Sur América, en Brasil, Uruguay, Venezuela y Argentina; en el sudeste asiático en Iran, Israel, Sri Lanka, y en Australia y Nueva Zelanda (Figura 2) (Guiry, M.D. \& Guiry, G.M, 2011). 


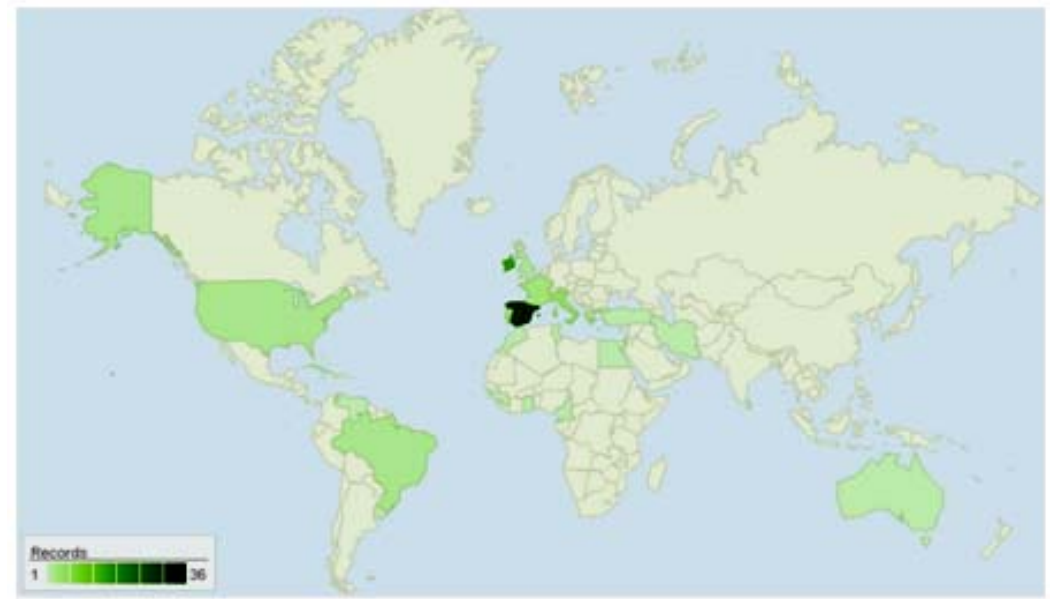

Figura 2. Distribución de Gigartina acicularis (Roth) J.V.Lamouroux, 1813 Fuente: Guiry, M.D. \& Guiry, G.M (2011)

Gigartina skottsbergii. Tiene un distribución sobre Sur América, donde se ha reportado en Argentina (Boraso \& Zaiso, 2011), en Chile (Buschmann, et al. 2001), en las Islas Malvinas (Wincke \& Clayton, 2002), y sobre Antartida y en algunas Islas Subantarticas (Figura 3) (Guiry, M.D. \& Guiry, G.M. 2011).

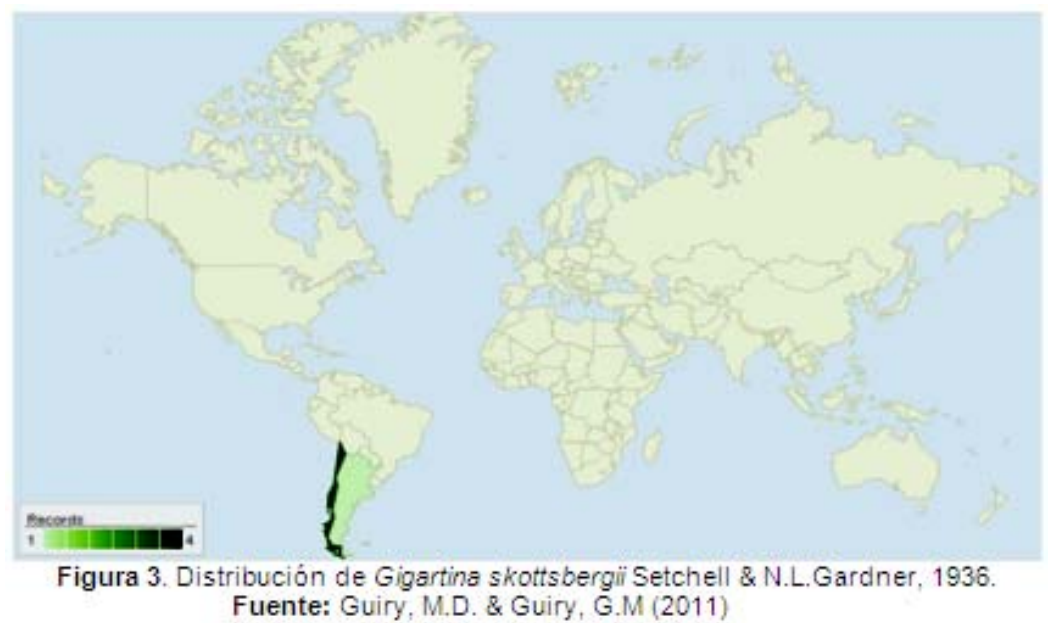

Gigartina pistillata. Se ha reportado en Europa, en Gran Bretaña, Francia, Grecia, Irlanda, Portugal, en las islas atlánticas como Azores, Islas Canarias y Madeira, también en nor-occidente africano en Maruitania, Morroco, Senegal (Figura 4) (Guiry, M.D. \& Guiry, G.M. 2011). 


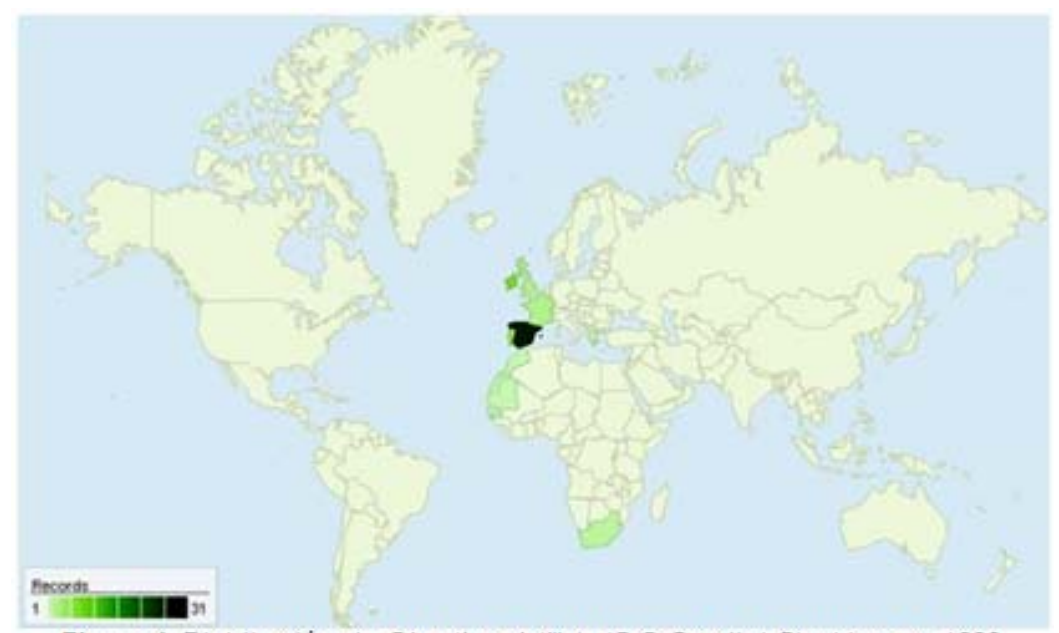

Figura 4. Distribución de Gigartina pistillata (S.G.Gmelin) Stackhouse, 1809. Fuente: Guiry, M.D. \& Guiry, G.M (2011)

\section{CICLO DE VIDA}

Corresponde a ciclo trifásico con alternancia de generaciones isomórfica (Figura 5) (Kim, 1976), que se caracteriza por presentar un gametofito y esporofito similares en morfología. Cada cistocarpo o soro tetrasporangial puede desarrollar miles de carposporas o tetrasporas respectivamente. La reproducción es del tipo oogámica donde la célula huevo es fecundada por un espermacio inmóvil. Después de la fecundación se produce la formación y desarrollo de los esporofitos, los cuales al madurar generan soros tetrasporangiales. (Piriz, 1996; Zamorano \& Westermeier, 1996), donde se puede presentar regeneración de tejidos por hapterios 0 por las mismas frondas. 


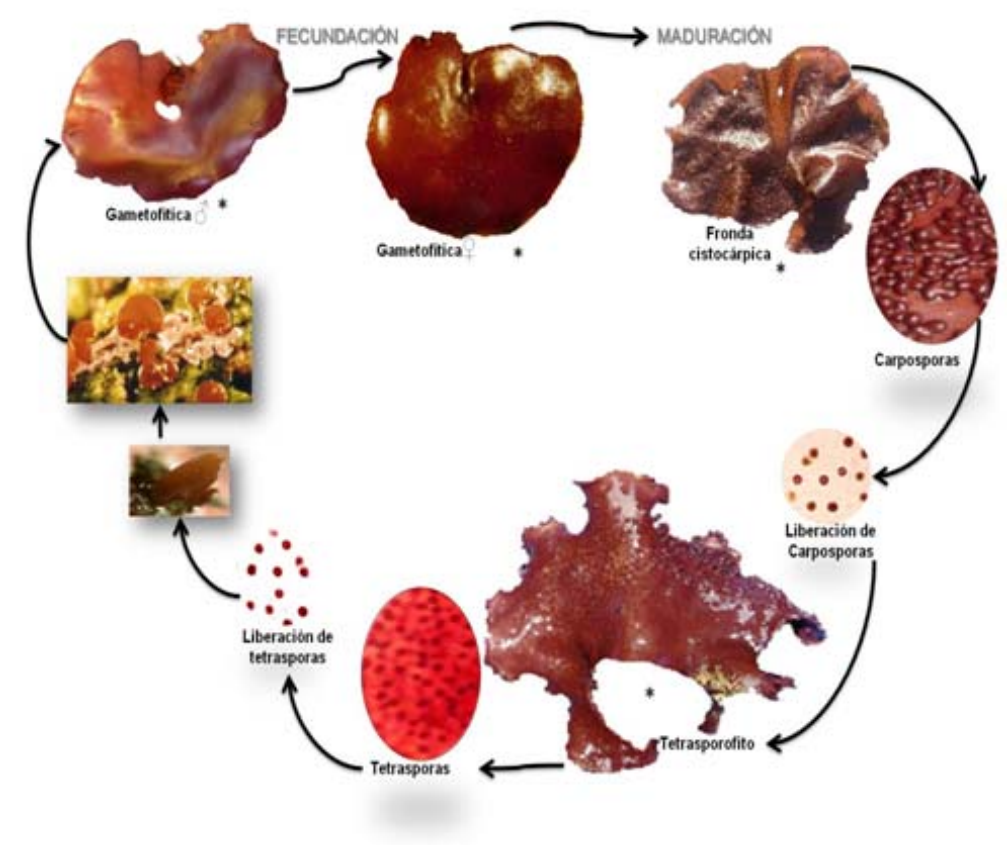

Figura 5. Diferentes etapas de desarrollo de Gigartina skottsbergü.

* Regeneración por hapterios o regeneración des de las frondas. Adaptado de Romo et al., 2001.

\section{CULTIVO}

El cultivo de Gigartina se desarrolla en dos fases (Figura 6): i) cultivo en laboratorio, ii) cultivo a mar abierto o repoblamiento.

Fase de laboratorio. Consiste en la inoculación de sustratos naturales o artificiales (cantos rodados o placas de cerámica), con esporas en condiciones semi-controladas en invernadero o en laboratorio. Las frondas reproductivas cistocarpicas y tetrasporicas silvestres son inducidas para la producción de esporas por secado a temperatura ambiente, alrededor de 2 horas para obtener la liberación de esporas. Buschman y colaboradores (2001) en su metodología para el cultivo de Gigartina skottsbergii reporta la siembra de Alícuotas de 360000 esporas $\mathrm{mL}^{-1}$ (tetrásporas y carpósporas) en placas de cerámica de $20 \mathrm{~cm}^{2}$ con crecimiento en tanques de vidrio de $50 \mathrm{~L}$ con $1,2 \mathrm{~m}$ de agua de mar filtrada, durante este experimento, la temperatura del agua osciló entre 12 y $15^{\circ} \mathrm{C}$, la salinidad varió de $30-32 \%$, pH 8 a 8,2 , y el fotoperiodo se mantuvo a las 12:12 (L:D), el crecimiento y supervivencia de los juveniles se registró fotográficamente utilizando un microscopio estereoscópico y fueron registradas mensualmente los cambios en la densidad (número de discos individuales por $\mathrm{cm}^{-2}$ ) y las tasas de crecimiento específicas. Una vez alcanzado el estado de desarrollo (después de 2-3 meses), los talos fijos a los sustratos fueron trasladados finalmente al mar, donde alcanzaron su talla comercial (fronda de $20 \mathrm{~cm}$ ) en un tiempo aproximado de 24 meses (Maschek, et al. 2011).

En esta etapa, el cultivo de Gigartina requiere manipulación especial para evitar la alta mortalidad de las cepas en el campo y 
favorecer la selección de las áreas de cultivo, donde la temperatura no puede superar los $14{ }^{\circ} \mathrm{C}$ (Araujo, et al. 2012), además de tener en cuenta que las bajas tasas de crecimiento de las especies de Gigartina, es primordial seleccionar linajes de crecimiento rápido, (Waaland, 1979.; Cheney, 1999.; Avila, 1999.; Buschmann, et al. 2004., Westermeier, et al. 2012).

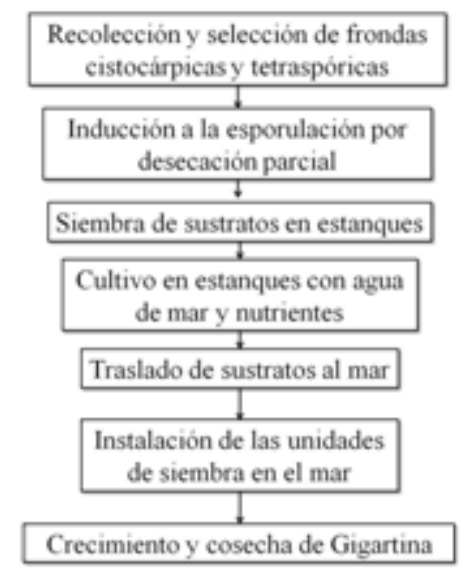

Figura 6. Esquema del cultivo de Gigartina Fuente: Autores.

Fase de cultivo a mar abierto. La repoblación por siembra directa consiste en sembrar sustratos artificiales o naturales usados en la etapa de desarrollo y germinación directamente en el mar con frondas reproductivas tetraspóricas y cistocárpicas (Westermeier, et al. 2012).

Para la repoblación por siembra directa Bussman y colaboradores (2001), se utilizaron como sustratos bloques de hormigón instaladas a una profundidad de $9.12 \mathrm{~m}$, simulando el sustrato natural para la fijación de las esporas. Artesanalmente, para tal proceso se cortan trozos de frondas reproductivas maduras (cistocárpicas y tetraspóricas) y se disponen sobre piedras de aproximadamente $30 \mathrm{~cm}^{2}$, las cuales son envueltas en una malla de algodón (alga y piedra), ubicándolas en el lecho marino a una densidad de 9 sustratos $/ \mathrm{m} 2$. La malla de algodón tiene un tiempo de biodegradación de 15 días, permitiendo el tiempo necesario para la fijación y desarrollo del disco o fronda. Después de la fijación, las plantas crecen hasta alcanzar su talla comercial (Vejar, 2009). El crecimiento de las especies de Gigartina es aproxiamdamente 30 meses para alcanzar un tamaño cosechable y comercial (caso de G. Skottsbergii) (Avila, 1999; Díaz \& Díaz, 2003).

Temperaturas óptimas de cultivo de algunas Gigartinas. Gigartina skottsbergii es una especies de agua fría, con un límite térmico de $5^{\circ} \mathrm{C}$ (Bischoff-Bäsmann \& Wiencke, 1996), es probable que dicho límite sea diferente para las poblaciones que se presentan en el extremo norte de la costa del Pacífico. Esto es confirmado por Buschmann y colaboradores (1999), quienes reportan mejores 
respuestas en la germinación a temperaturas iguales a $5^{\circ} \mathrm{C}$ (Buschmann, et al. 2004.; Eggert, 2012).

Gigartina acicularis es un alga perenne relativamente rara de las Islas Británicas (Guiry, 1984). Se desarrolla en el intermareal inferior y el submareal de poca profundidad en lugares expuestos o semiprotegidos asociados frecuentemente con limo o arena fina. La especie se encuentra desde las Islas Británicas al sur de Camerún, Carolina del Norte a Uruguay, y en el Mediterráneo oriental; forma gametangios en cultivo a una temperatura de $14-18{ }^{\circ} \mathrm{C}$ (Guiry \& Cunningham, 1984, Hommersand, et al. 1992).

Pacheco y colaboradores (1992), registraron tasas de crecimiento máximo $(10,6 \%$ día-1) de Gigartina pectinata a finales de la primavera, donde el crecimiento en las placas de gradiente de luztemperatura era óptima en irradiaciones inferiores a las encontradas en el campo y en las temperaturas de $14-26{ }^{\circ} \mathrm{C}$; También, los autores concluyeron que el crecimiento en el campo, así como en el laboratorio se redujo fuertemente a temperaturas mayores de $26{ }^{\circ} \mathrm{C}$ y bajo altas densidades de flujo de fotones.

\section{APRECIACIONES ECONÓMICAS}

La principal restricción para la promoción del desarrollo de Gigartina es su bajo potencial de crecimiento. Es importante evaluar el potencial de crecimiento de las algas para aumentar la producción cuando se desarrolla un programa de selección de cepas. En este contexto, la tasa de crecimiento, y la producción potencial en Gigartina requiere más atención e igual al desarrollo de un programa de selección de las cepas en los procesos de cultivo. La propagación vegetativa de dicha especie es una característica que contribuiye a la creación de dicho programa (Buschmann, et al. 1999) como parte de tal estrategia, se están realizando investigaciones para obtener variedades con mayores tasas de crecimiento.

Las principales especies de explotación comercial (alimenticio y farmacéutico) en el mundo son Gigartina Skottsbergii, G. pistillata y G. teedii (Figura 1). El cultivo de G. skottsbergii en Chile se encuentra limitada por su lento crecimiento y los valores de mercado. Al igual que con otros productores de gel de algas, los precios son bastante bajos, y en el caso de G. skottsbergii el precio pagado a los productores de EE.UU., ha fluctuado entre 0,7 dólares y 0,9 dólares EE.UU*kg-1 de peso seco en el pasado (19981999).(Buschman, et al.; 2001; Pereira \& Van de Velde, 2011)

\section{PRECURSORAS DE CARRANGENANOS}

Carragenanos son los terceros hidrocoloides más importantes de la industria de alimentos, después de gelatina (origen animal) y el almidón (Van de Velde \& de Ruiter, 2002). Los carragenanos son polisacáridos de alto valor comercial presentes en las paredes 
celulares y espacios intercelulares de Rhodophyta que pertenecen a la Gigartinales (McCandless, 1979.; McHugh, 2003; Dhargalkar \& Verlecar, 2009; Usov, 2011, Díaz, et al. 2012).

Existen cuatro tipos de ficocoloides de importancia comercial: Kappa I, Kappa II, lota y Lambda (Pereira, et al. 2009-2011; Hernández-González, et al. 2007; Kumar, et al. 2012). Estevez y colaboradores (2003) sugieren una correspondencia directa entre ploidía (haploide/diploide) y tipo de carragenano biosintetizado (kappa/lambda), por lo tanto, cada especie de alga produce carrageninas características (Tabla 3 ), de las cuales se ha reportado que Gigartina skottsbergii produce carragenina de la familia Kappa en la fase gametofítica y carragenanos Lambda en la fase esporofítica (Piriz, 1996.; Avila, et al., 1999), en proporciones de 5,09 ppm (kappa), 5,29 ppm (lota), 5,55 ppm (Lambda), 5.49 (Xi),5,30 ppm (Piruvato) y 5.35 (almidón) (Pereira, et al. 2011), mientras que, los ficocoloides de G. teedii se consideran híbridos resultantes de la carragenina, fragmentos yuxtapuestos de Kappa e lota (Zinoun, et al. 1993) y Gigartina pistillata produce carrageninas de la familia de Kappa (híbrido Kappa/lota/mu/nu) (Amimi, et al. 2001; Pereira, et al. 2009), así también G. stellata (Rudolf, 2000; Datta, et al. 2010), entonces el cambio biosintético, durante el ciclo biológico, de Kappa a Lambda carragenanos y de lambda a kappa carragenanos puede producirse después de la liberación y desarrollo de las esporas (carposporas y tetrasporas, respectivamente) (Van de Velde, 2008; Díaz, et al. 2012).

Tabla 3. Tipos de carragenanos producidos por al gunas especies de Gigartina, teniendo en cuenta la fase del ciclo de vida

\begin{tabular}{|c|c|c|c|}
\hline Familia & Especie & $\begin{array}{l}\text { Fase del ciclo de } \\
\text { vida }\end{array}$ & Carrangenano \\
\hline \multirow{8}{*}{ Gigartinaceae } & Gigartina pistillata & Talo no fructifico & Kappa -lota (mu/nu) \\
\hline & G. Pistillata & Tetrasporofito & Xi-Lambda \\
\hline & G. Pistillata & Talo heterospórico & Xi-Lambda \\
\hline & G. Pistillata & $\begin{array}{l}\text { Gametofito } \\
\text { femenino }\end{array}$ & Kappa -lota (mu/nu) \\
\hline & G. Pistillata & $\begin{array}{l}\text { Talo heterospórico } \\
\underline{Q}\end{array}$ & Kappa -lota (mu/nu) \\
\hline & Gigartina skottsbergii & Gametofítica & Kappa \\
\hline & G. skottsbergii & Esporofitica & Lambda \\
\hline & Gigartina. teedil & Gametofitica & Kappa-lota \\
\hline
\end{tabular}

\section{TOXICIDAD}

La eficacia y la toxicidad potencial de los recursos marinos en la medicina popular han sido evaluados científicamente, es así como Bussmann y colaboradores (2011), en su estudio sobre concentración media letal de las plantas con uso medicinal en Perú, consideraron como no toxico el extracto acuoso de Gigartina sp. (> $10.000 \mu \mathrm{g} / \mathrm{ml}$ LC50) (Vo, et al. 2011).

OTROS USOS 
Ha sido determinada la actividad antiviral en Gigartina tenella (Ohata, et al. 1998), por la presencia de Sulquinovosildiacilglicerol, KM043 301, un nuevo sulfolipido KM043, que pertenece a la 6-sulf$\mu$-D-quinovopiranosil (1 $\rightarrow$ 3')-1'2'-diacilglicerol (SQDG) clase de compuestos que ha sido aislado de esta especie de alga roja como un potente inhibidor del ADN eucariota y el VIH-I de la transcriptasa inversa de tipo 1.

Similares reportes hace Carlucci y colaboradores $(1997,1999)$ sobre el efecto antiviral de las carrageninas, los notaron que la $\lambda$ carragenina y la parcialmente ciclada $\mu /$ iota carragenina obtenida a partir de Gigartina skottsbergii tiene potenciales efectos antivirales contra HSV tipos 1 y 2, durante la etapa de adsorción del virus. También se ha identificado un tipo de polisacárido sulfatado (SP), como precursor de actividad anticoagulante en forma de galactano sulfatado extraído de la carragenina de G. skottsbergii (Carlucci, et al. 1997.; Wijesekara et al. 2010.; Rajapakse \& Kim, 2011.; Samaraweera, et al. 2012).

\section{BIBLIOGRAFÍA}

- Amimi, A.; Mouradi, A.; Givernaud, T.; Chiadmi, N.; \& Lahaye, M. (2001). Structural analysis of Gigartina pistillata carrageenans (Gigartinaceae, Rhodophyta). Carbohydrate Research, 333(4): 271-279.

- Araujo, R.; Arenas, F.; Åberg, P.; Sousa-Pinto, I. \& Serrão, E. A. (2012). The role of disturbance in differential regulation of co-occurring brown algae species: Interactive effects of sediment deposition, abrasion and grazing on algae recruits. Journal of Experimental Marine Biology and Ecology, 422-423 (0): 1-8.

- Avila, M.; Candia, A.; Nuñez, M. \& Romo, H. (1999). Reproductive biology of Gigartina skottsbergii (Gigartinaceae, Rhodophyta) from Chile. Hydrobiologia 398/399: 149-157.

- Bischoff-Bäsmann, B. \& C. Wiencke. (1996). Temperature requirements for growth and survival of Antarctic Rhodophyta. J.Phycol. 32: 525-535.

- Bold, H.C.; Wynne, M.J. (1985). Introduction to the algae structure and reproduction, second ed., Prentice-Hall Inc., Englewood Cliffs, NJ, 07632, pp. 1-33.

- Buschmann, A.; Correa, J \& Westermier, R. (1999). Recent advances in the undertandisng of biological basis for Gigartina skottsbergii (Rhodophyta) cultivation in Chile. Hydrobiologia 398/399: 427-434.

- $\quad$, Correa, J.; Westermeier, R.; Paredes, M.; Aedo, D.; Potin, P.; Aroca, G.; Beltrán, J \& Hernandez, M. (2001). Cultivation of Gigartina Skottsbergii (Gigartinales, Rhodophyta): Recent advances and 
challenges for future. Journal of Applied Phycology. 13: 255-266.

- Buschmann, A. H.; Correa, J. A.; Westermeier, R.; Hernández-González, M. a. d. C. \& Norambuena, R. (2001). Red algal farming in Chile: a review. Aquaculture, 194 (3-4), 203-220.

H.; Varela, D.; Cifuentes, M.; del Carmen Hernández-González, M.; Henríquez, L.; Westermeier, R. et al. (2004). Experimental indoor cultivation of the carrageenophytic red alga Gigartina skottsbergii. Aquaculture, 241(1-4), 357-370.

- Bussmann, W. G.; Malca, A.; Glenn, D.; Sharon, B.; Nilsen, B.; Parris, D.; Dubose, D.; Ruiz, J.; Saleda, M.; Martinez, L.; Carillo, K.; Walker, A.; Kuhlman \& A. Townesmith. (2011). "Toxicity of medicinal plants used in traditional medicine in Northern Peru." Journal of Ethnopharmacology 137(1): 121-140.

- Carlucci, M.J.; Pujol, C.A.; Ciancia, M.; Noseda, M.D.; Matulewicz, M.C.; Damonte, E.B.; Cerezo, A.S. (1997). Antiherpetic and anticoagulant properties of carrageenans from the red seaweed Gigartina skottsbergii and their cyclized derivatives: correlation between structure and biological activity. Int.J. Biol. Macromol 20:97-105.

- Ciancia, M.; Matulewicz, M.C.; Cerezo, A.S.; Damonte, E.B. (1999). Antiherpetic activity and mode of action of natural carrageenans of diverse structuraltypes. Antivir. Res. 43: 93-102.

- Cheney, D.P. (1999). Strain improvement of seaweeds through genetic manipulation: current status. World Aquaculture: 30: 55-56; 66-67.

- Datta, S.; K. Mody, G.; Gopalsamy \& B. Jha. (2010). Novel application of $\hat{i}^{\circ}$-carrageenan: As a gelling agent in microbiological media to study biodiversity of extreme alkaliphiles. Carbohydrate Polymers 85(2): 465-468.

- Dhargalkar, V.K., Verlecar, X.N., 2009. Southern Ocean seaweeds: a resource for exploration in food and drugs. Aquaculture 287. 229-242

- Díaz, O.; Tapia, Y.; Muñoz, O.; Montoro, R.; Velez, D. \& Almela, C. (2012). Total and inorganic arsenic concentrations in different species of economically important algae harvested from coastal zones of Chile. Food and Chemical Toxicology, 50(3-4). 744-749.

- Díaz-Pulido, G \& Díaz-Ruíz. (2003). Diversity of benthic marine algae of the Colombian Athlantic. Biota Colombiana. 4 (2): 203-246.

- Eggert, A. (2012). Seaweed Responses to Temperature Seaweed Biology. In C. Wiencke \& K. Bischof (Eds.), Vol. 219. pp. 47-66: Springer Berlin Heidelberg.

- El Gamal, A. A. (2010). Biological importance of marine algae.. Saudi Pharmaceutical Journal, 18(1), 1-25.

- Estevez, J.; Ciancia, M \& Cerezo. A. (2003). Biosíntesis de carragenanos durante el ciclo de vida del alaga roja 
comercial Gigartina Skottsbergii (Gigartinaceae, Rhodophyta). Bol. Soc. Argent. Bot. 38 (supl.): p.152

- Guiry, M.D. \& Cunningham, E.M. (1983). Life history and hybridization in culture of Gigartina acicularis (Rhodophyta) from England and France. British Phycological Journal 18: 204 (abstract)

- Guiry, M.D. (1984). Photoperiodic and temperature responses in growth and tetrasporogenesis of Gigartina acicularis (Rhodophyta) from Ireland. Helgoländer Meeresunters, 38: 335-347.

- Guiry, M.D. \& Guiry, G.M. (2011). AlgaeBase. World-wide electronic publication, National University of Ireland, Galway. http://www.algaebase.org. searched on 10 November 2011.

- Hernández-Gonzáles, M.; Buschman, A.; Cifuentes, M.; Correa, J.; Westermeier, R. (2007). Vegetative propagation of the carrageennophytic red alga Gigartina skottsbergii Setchell et Gardner: Indoor and field experiements. Aquaclture 262: 120-128.

- Hommersand, M., Fredericq, S., \& Cabioch, J. (1992). Developmental morphology of Gigartina pistillata (Gigartinaceae, Rhodophyta). Phycologia, 31(3/4), 300325.

- Kim, D.H., (1976). A study of the development of cystocarps and tetrasporangial sori in Gigartinaceae, (Rhodophyta, Gigartinales). Nova Hedwigia 27: 1-146.

- Kumar, S.; Godiya, C.B. \& Siddhanta, A. K. (2012). Carrageenan from Sarconema scinaioides (Gigartinales, Rhodophyta) of Indian waters. [doi: 10.1016/j.carbpol.2011.09.062]. Carbohydrate Polymers, 87(2), 1657-1662.

- Maschek, J. A.; Bucher, C. J.; Olphen, A.; Amsler, C. D.; McClintock, J. B. \& Baker, B. J. (2011). The Pursuit of Potent Anti-influenza Activity from the Antarctic Red Marine Alga The Biological Activity of Phytochemicals. In D. R. Gang (Ed.) Vol. 41. pp. 1-12. Springer New York.

- McCandless, E.L.; Craigie, J.S. (1979) Sulfated polysaccharides in red and brown algae. Ann. Rev. Plant.Physiol. 30: 41- 53.

- McHugh, D.J. (2003) A guide to the seaweed industry. FAO Fish Biol Tech Pap 441:52-72

- Monte-Luna, P.; Lluch-Belda, D.; Carmona, R.; ReyesBonilla, H.; Castro-Aguirre, J.; Serviere-Zaragoza, E. (2008). Extinciones en el mar: Mitos $Y$ realidades. Interciencia, 33(1): 74-80.

- Ohata, K.; Mizushina, Y.; Hirata, N.; Sugawara, F.; Mutsukage, A.; Yoshida, S.; Sakaguchi, K. (1998). Sulphoquinovosy-ldiacylglycerol, KM043 a new potent inhibitor of eukaryotic DNA polymerases and HIV-reverse transcriptase type from a marine red alga Gigartina tenella. Chem. Pharm. Bull. 46: 684-686. 
- Pacheco, I., Zertuche, J., Cabello, A \& Brinkhuis, B. 1992. Growth responses and seasonal biomass vriation of Gigartina pectinata Dawson (Rhodophyta) in the gulf of California. Journal of experimental Marine Biology and Ecology. 157 (2): 263-274

- Pereira, L.; Critchley, A.; Amado, A.; Ribeiro-Claro, P. (2009). A comparative analysis of phycocolloids produces by underutilized versus industrially utilized carrageenophytes (Gigartinales, Rhodophyta). J. Appl. Phycol. 21: 599-605

- Pereira, L. \& Van de Velde, F. (2011). "Portuguese carrageenophytes: Carrageenan composition and geographic distribution of eight species (Gigartinales, Rhodophyta)." Carbohydrate Polymers 84(1): 614-623.

- Piriz, M. L. (1996). Phenology of a Gigartina skottsbergii Setchell et Gardner population in Chubut Province (Argentina). Bot. mar. 39: 311-316.

- Rajapakse, N. \& Kim, S.-K. (2011). Nutritional and Digestive Health Benefits of Seaweed. In K. Se-Kwon (Ed.). Advances in Food and Nutrition Research. Volume 64, pp. 17-28. Academic Press.

- Romo, H.; Avila, M \& Candia, A. (2001). Manual de técnicas de cultivo y repoblación de "luga roja" (Gigartina skottsbergii). Proyecto FONDEF D97I1064. IFOPUniversidad de Concepción, Chile. pp. 32

- Rudolph, B. (2000). Seaweed product: Red algae of economic significance, R.E. Martin, E.P.; Carter, L.M.; Davis, G.J.; Flich (Ed.). Marine and freshwater products handbook, Technomic Publishing Company Inc, Lancaster, USA. pp. 515-529

- Samaraweera, A. M.; Vidanarachchi, J. K. \& Kurukulasuriya, M. S. (2011). Industrial Applications of Macroalgae Handbook of Marine Macroalgae. pp. 500-521. John Wiley \& Sons, Ltd.

- Usov, A. I. (2011). Polysaccharides of the red algae. In $\mathrm{H}$. Derek (Ed.), Advances in Carbohydrate Chemistry and Biochemistry. Vol. 65, pp. 115-217. Academic Press.

- Van de Velde, F.; de Ruiter G.A. (2002). Carrageenan. In: Vandamme, E.J.; Baets, S.D.; Steinbèuchel A (Eds) Biopolymers $\vee 6$ polysaccharides II. Polysaccharides from eukaryotes. Wiley, Weinheim. pp 245-274.

- (2008). Structure and function of hybrid carrageenans. Food Hydrocolloids, 22(5), 727-734.

- Vejar, P. (2009). Evaluación técnica y económica para el desarrollo de acuicultura en áreas de manejo y explotación de recursos bentónicos en la región de Lagos. Trabajo de grado. Universidad Austral de Chile. Escuela de acuicultura y Pesquerías. p. 91

- Vo, T.S.; Ngo, D.H.; Ta, Q. V. \& Kim, S.-K. (2011). Marine organisms as a therapeutic source against herpes simplex virus infection. European Journal of Pharmaceutical Sciences, 44(1-2), 11-20. 
- Waaland, J.R. (1979). Growth and strain selection in the marine alga Gigartina exasperata (Florideophyceae). Int. Seaweed Symp. 9: 241-247.

- Westermeier, R.; Patiño, D.; Murúa, P.; Quintanilla, J.; Correa, J.; Buschmann, A. et al. (2012). A pilot-scale study of the vegetative propagation and suspended cultivation of the carrageenophyte alga \&lt;i\&gt;Gigartina skottsbergii\&lt;/i\&gt; in southern Chile. Journal of Applied Phycology. 24(1), 11-20.

- Wijesekara, I. R.; Pangestuti \& S.-K. Kim. (2010). "Biological activities and potential health benefits of sulfated polysaccharides derived from marine algae." Carbohydrate Polymers 84(1): 14-21.

- Zamorano, J. \& R. Westermeier, (1996). Phenology of Gigartina skottsbergii (Gigartinaceae, Rhodophyta) in Ancud Bay, southern Chile. Hydrobiologia, 326/327: 253258.

- Zinoun, M.; Cosson, J \& Deslandes, E. (1993). Physicochemical characterization of carrageenan from Gigartina teedii (Rooth) Lamouroux (Gigartinales, Rhodophyta). Journal of Applied Phycology 5: 23-28

1. Bióloga, M.Sc. en Recursos Hidrobiológicos Continentales. Instituto de Postgrados. Facultad de Ciencias Naturales, Exactas y de la Educación. Grupo de Estudios Ambientales (GEA). Departamento de Biología. Universidad del Cauca mdsalazar@unicauca.edu.co

2. Biólogo, Universidad del Cauca. Popayán, Colombia. salazarbio@gmail.com 